\title{
Cartesian contrivances
}

If matter fills the Universe, making everything happen by its interactions, what does it all look like? René Descartes may have been over-mechanistic in his view, but his efforts to visualize the invisible created striking images.

\section{Martin Kemp}

$\mathrm{H}$

ow to envisage and represent the ways hat nature works has been the major challenge for illustrators of physical phenomena. Such things as invisible forces or the mechanisms responsible for 'action at a distance' can be described abstractly in words or encapsulated in mathematical formulas. But generations of natural philosophers and physicists have felt an apparently compelling need to develop and express their theories in more concrete terms, often by reference to existing machines or specially contrived mechanical analogies.

For a philosopher as obsessed with mechanism as René Descartes, the relationship between 'seeable' machines and the unseen machinery of God's cosmos was a matter of the greatest moment.

"The operations of things made by skill are, for the most part, performed by apparatus large enough to be easily perceived by the senses: for this is necessary so that they can be made by men. On the other hand, natural effects always depend on some devices so minute that they escape all senses."

To overcome this problem, Descartes' publications brilliantly exploit virtually all

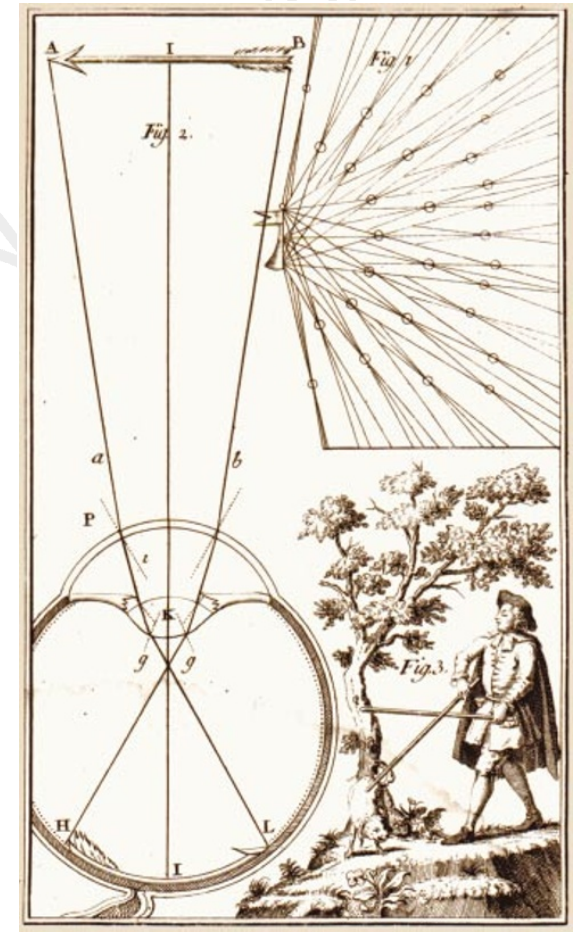

Illustration of the optics of the eye and Descartes' crossed sticks analogy, from A Physical Essay on the Senses by Claude Nicolas le Cat, 1750.

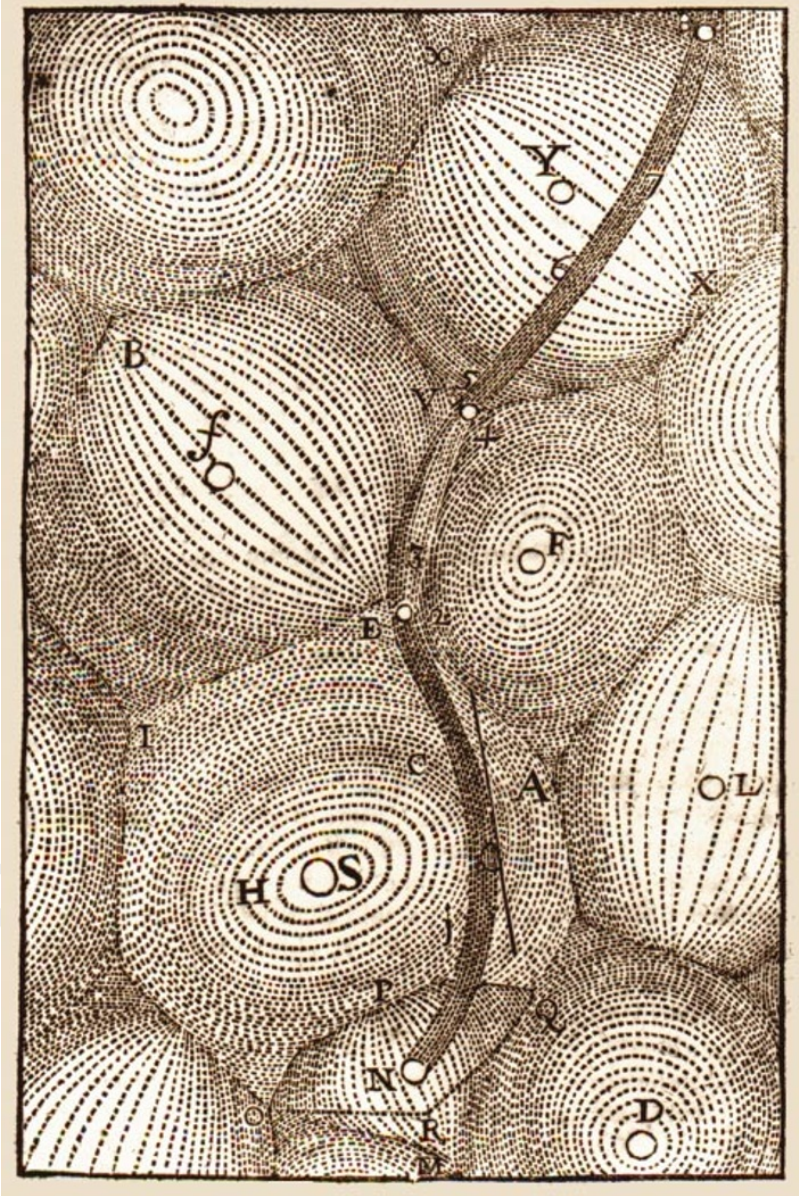

Descartes' "celestial
vortices", from Principia

Philosophiae, 1644. the modes of illustration available in the seventeenth century, ranging from pictorial representations to abstract diagrams.

\section{He exhibited a particular genius for} demonstrating invisible phenomena in terms of mechanical contrivances.

A number of his visual conceptions, such as his analysis of binocular vision and the inverted image on the retina in terms of a blind man with a pair of sticks, became oftrepeated classics.

Descartes was aware, however, that demonstration by mechanical analogy could be taken too literally - that the behaviour of the contrivance could all too easily be seen as constituting proof of what is actually happening with the phenomenon. Even for a philosopher who sought to explain all aspects of the physical world in terms of the action of matter on matter, illustration by mechanical analogy was a limited device.

He scolded those who viewed his aids to visualization in too obvious a way: "I did not say that light was extended like a stick, but like the actions or movements transmitted by a stick". The blind man probing with his sticks simply helps us to understand how vision might work in terms of the underlying properties of things in action. The phenomenon and its mechanical visualization were analogous symptoms of the prime properties of magnitude, figure and motion.

His desire to convey the physical texture of even the most theoretical of his speculations is vividly shown in the 'picture' of his famous celestial vortices. The analogy here is hydraulic. Between the extremes of the most refined and luminous kinds of particulate matter and the coarse, weighty material of the Earth and planets were mobile globules that flowed in ceaseless whirlpools. These constituted a dynamic kind of cosmic foam that activated the apparent voids between celestial bodies. His extraordinary illustration of the Sun in the midst of its own vortex, packed within a three-dimensional system of contiguous vortices, stretches even his illustrative resources to breaking point. But it still does a vital job in a way that words could not emulate.

Martin Kemp is in the Department of the History of Art, University of Oxford, 35 Beaumont Street, Oxford OX1 2PG, UK. 